\title{
Transcriptome Analysis of Early Responsive Genes in Rice during Magnaporthe oryzae Infection
}

Yiming Wang ${ }^{1, \uparrow}$, Soon Jae Kwon ${ }^{2, \uparrow}$, Jingni Wu ${ }^{1}$, Jaeyoung Choi $^{3}$, Yong-Hwan Lee ${ }^{3}$, Ganesh Kumar Agrawal ${ }^{4,5}$, Shigeru Tamogami ${ }^{6}$, Randeep Rakwal ${ }^{4,5,7}$, Sang-Ryeol Park ${ }^{8}$, Beom-Gi Kim ${ }^{8}$, Ki-Hong Jung ${ }^{9}$, Kyu Young Kang ${ }^{10}$, Sang Gon Kim ${ }^{10 *}$ and Sun Tae Kim ${ }^{2 *}$

${ }^{1}$ Department of Plant Microbe Interaction, Max Planck Institute for Plant Breeding Research, Carl-von-Linné-Weg 10, 50829 Köln, Germany

${ }^{2}$ Department of Plant Bioscience, Life and Industry Convergence Research Institute, Pusan National University, Miryang 627-706, Republic of Korea

${ }^{3}$ Department of Agricultural Biotechnology, Center for Fungal Genetic Resources and Center for Fungal Pathogenesis, Seoul National University, Seoul 151-921, Republic of Korea

${ }^{4}$ Research Laboratory for Biotechnology and Biochemistry (RLABB), GPO Box 13265, Kathmandu, Nepal

${ }^{5}$ GRADE Academy Pvt. Ltd., Adarsh Nagar-13, Main Road, Birgunj, Nepal

${ }^{6}$ Laboratory of Biologically Active Compounds, Department of Biological Production, Akita Prefectural University, Akita 010-0195, Japan

${ }^{7}$ Organization for Educational Initiatives, University of Tsukuba, Tsukuba 305-8577, Ibaraki, Japan

${ }^{8}$ Molecular Breeding Division, National Academy of Agricultural Science, RDA, Suwon 441-707, Republic of Korea

${ }^{9}$ Graduate School of Biotechnology and Crop Biotech Institute, Kyung Hee University, Yongin 446-701, Republic of Korea

${ }^{10}$ Plant Molecular Biology and Biotechnology Research Center/Division of Applied Life Science (BK21 Program), Gyeongsang National University, Jinju 660-701, Republic of Korea

(Received on July 4, 2014; Revised on July 25, 2014; Accepted on July 30, 2014)

Rice blast disease caused by Magnaporthe oryzae is one of the most serious diseases of cultivated rice (Oryza sativa L.) in most rice-growing regions of the world. In order to investigate early response genes in rice, we utilized the transcriptome analysis approach using a $300 \mathrm{~K}$ tilling microarray to rice leaves infected with compatible and incompatible $M$. oryzae strains. Prior to the microarray experiment, total RNA was validated by measuring the differential expression of rice defenserelated marker genes (chitinase 2, barwin, $P B Z 1$, and $P R-10)$ by RT-PCR, and phytoalexins (sakuranetin and momilactone A) with HPLC. Microarray analysis revealed that 231 genes were up-regulated $(>2$ fold change, $p<0.05$ ) in the incompatible interaction compared to the compatible one. Highly expressed genes

\footnotetext{
"These authors contributed equally to this work.

*Co-corresponding authors.

Sun Tae Kim

Phone) +82-55-350-5505, FAX) +82-55-350-5509

E-mail) stkim71@pusan.ac.kr

Sang Gon Kim

Phone) +82-55-772-1961, FAX) +82-55-772-1969

E-mail) sen600@hanmail.net
}

were functionally characterized into metabolic processes and oxidation-reduction categories. The oxidative stress response was induced in both early and later infection stages. Biotic stress overview from MapMan analysis revealed that the phytohormone ethylene as well as signaling molecules jasmonic acid and salicylic acid is important for defense gene regulation. WRKY and Myb transcription factors were also involved in signal transduction processes. Additionally, receptor-like kinases were more likely associated with the defense response, and their expression patterns were validated by RT-PCR. Our results suggest that candidate genes, including receptor-like protein kinases, may play a key role in disease resistance against $M$. oryzae attack.

Keywords : defense response, Magnaporthe oryzae, MapMan analysis, rice, transcriptomics

Rice blast fungus (Magnaporthe oryzae), known as a hemibiotrophic fungal pathogen, is the causative agent of rice blast disease (Couch et al., 2005; Talbot, 2003). After adhesion of spores onto the leaf surface, M. oryzae initiates 
germination of a germ tube within $2 \mathrm{~h}$, forming a special infection-related structure called an 'appressorium' in the very early stages $(2-20 \mathrm{~h})$ of interaction (Ribot et al., 2008). Melanized appressoria can be generated by high pressure, which could help fungi to penetrate plant cell walls with high physical forces (Ribot et al., 2008). Initiation of an early interaction between rice and rice blast fungus starts in the penetration stage and lasts until the early biotrophic growth stage, which occurs within 48 hour postinoculation (hpi).

Previous transcriptome studies by using microarrays or RNA-seq analysis have attempted to understand interaction between rice and rice blast fungus. Wei and colleagues employed microarray analysis to study transcriptome changes in compatible and incompatible rice cultivars at $24 \mathrm{hpi}$ after rice blast fungus infection (Wei et al., 2013), suggesting that the transcriptional profiles of rice in compatible and incompatible interaction are mostly similar. The transcription factor gene WRKY47, which was identified in their study, was overexpressed in transgenic rice and resulted in increased resistance to rice blast fungus (Wei et al., 2013). In another similar RNA-seq experiment, gene ontology (GO) enrichment in compatible and incompatible interaction remained similar, whereas the genes sets contributing to each GOs were dissimilar (Bagnaresi et al., 2012). Moreover, in compatible interaction, genes related with phytoalexin biosynthesis, flavin-containing monooxygenase, chitinase, and glycosyl hydrolase 17 were dramatically up-regulated (Bagnaresi et al., 2012). More recently, an RNA-seq-based transcriptome analysis showed different transcriptional regulation in both rice and rice blast fungus (Kawahara et al., 2012). More drastic changes were observed in incompatible interaction compared with compatible one at $24 \mathrm{hpi}$ in both rice and blast fungus in this stage. Further, several fungal genes encoding secreted effectors, which may be involved in the initial infection process, were up-regulated. These studies provided clues for understanding the rice immune response against rice blast fungus attack as well as how $M$. oryzae counters host defense.

Due to co-evolution processes, rice have developed molecular mechanisms to suppress successful infection by pathogens, such as recognition of pathogens through receptors, generation of reactive oxygen species (ROS), expression of pathogenesis-related (PR) proteins, and accumulation of anti-microbial secondary defense compounds, termed phytoalexins (Jwa et al., 2006). To recognize pathogen infection, plants contain a group of receptor-like kinases (RLK), which can bind with conserved microbeassociated molecular pattern (MAMP) ligands (Jones and
Dangl, 2006). Interaction between receptors and MAMPs, such as FLS2 and flg22, trigger activation of kinase domains and signal transduction through phosphorylation (Chinchilla et al., 2007). In response to pathogen attack, ROSs are rapidly produced and transmitted to engage immune function, such as the hypersensitive response (Frederickson Matika and Loake, 2014). Meanwhile, ROS also play an important role in cell signaling and homeostasis. ROS-detoxifying enzymes are highly accumulated in the anti-pathogen infection process and help balance redox changes inside cells (Pang et al., 2011). PR genes are those genes that are involved in the plant defense response, and they function in suppressing pathogen infection, such as thaumatin-like proteins and chitinases (Sels et al., 2008). Overexpression of those defense related genes in plants has shown the ability to increase resistance to fungal infection (Datta et al., 1999; Iqbal et al., 2012; Wu et al., 2013). Therefore, transcriptome analysis of genes involved in the recognition of pathogen signaling as well as investigation of plant defense-related genes at early and late time points are both important for understanding plant immune responses to biotic stresses.

In this study, we performed a comparative transcriptome analysis of rice and rice blast fungus interaction in early and later interaction stages. Rice leaves infected with incompatible and compatible fungal strains at 12 and 48 hpi were used in our study. A total of 608 genes showed statistically significant changes in this process, and they were found to have multiple biological functions, such as cell signaling, redox states, and proteolysis. We also identified 18 genes encoding receptor-like kinases and validated them by RT-PCR, which showed good correlation with the microarray data. Our study is helpful for understanding rice$M$. oryzae interaction, in both early and later stages of the infection.

\section{Materials and Methods}

Plant growth condition and pathogen inoculation. Fourth- to fifth-leaf-stage rice seedlings (Oryzae sativa cv. Jinheung) grown under natural light in a greenhouse (20$30^{\circ} \mathrm{C}$ ) were used for inoculation of rice blast fungus. For fungal inoculation, a conidial suspension $\left(1 \times 10^{5}\right.$ conidia/ $\mathrm{mL}$ ) of $M$. oryzae race KJ401 and KJ301 (incompatible and compatible, respectively, to cv. Jinheung) was sprayed onto the leaves using an air sprayer. Inoculated plants were kept in a humidity chamber at $28^{\circ} \mathrm{C}$ and harvested at 12 , 24, 48, and 72 hpi. For transcript profiling, leaf samples were collected at $12,24,48$, and $72 \mathrm{hpi}$, were frozen in liq- 
uid nitrogen, and stored at $-70^{\circ} \mathrm{C}$.

RNA isolation and RT-PCR. Total RNA was isolated from rice leaves (infected and corresponding control) using TRIzol RNA isolation reagent (Invitrogen, Paisley, UK) according to the manufacturer's instructions and dissolved in ribonuclease-free water. Total RNA samples $(5 \mu \mathrm{g}$ per reaction) were reverse-transcribed using a cDNA synthesis system for RT-PCR according to the manufacturer's instructions (Invitrogen, Madison, WI, USA). RT-PCRs were performed according to the method reported earlier (Kim et al., 2009). Primers used for RT-PCR experiments are listed (Supplementary Table 1). Beta-actin, eEFla, and OsUbi5 transcripts were used as an internal control to normalize not only the concentration of cDNA in each sample, but also the gene expression profile over the time course.

Measurement of phytoalexins. Infected rice leaves (100 $\mathrm{mg}$ ) were extracted with $80 \%$ methanol by boiling for 5 min. Three microliters of the crude extract was then injected onto an HPLC and analyzed by LC-MS/MS according to previously reported conditions (Tamogami and Kodama,
2000). TSQ Quantum Ultra-MS/MS equipped with Accela 600 HPLC system (Thermo Fisher Scientific Inc. MA, USA) and a reversed phase column (Waters Atlantis T3, 3 $\mu \mathrm{m}, 2.1 \times 15 \mathrm{~mm}$, flow rate of $0.2 \mathrm{ml} / \mathrm{min}$ with $80 \%$ aqueous methanol) was used. Sakuranetin and momilactone A were monitored at combinations of $\mathrm{m} / \mathrm{z} 287 / 167$ and $\mathrm{m} / \mathrm{z}$ 315/271 in MRM (multiple reaction monitoring) modes by APCI (Atmospheric Pressure Chemical Ionization) method according to the previous method with slight modifications (Tamogami and Kodama, 2000).

Microarray hybridization, data processing, and statistical analysis. The average size of probe was 60 nucleotides with its Tm value adjusted from 75 to $85^{\circ} \mathrm{C}$. The microarray was manufactured at NimbleGen Inc (http:// www.nimblegen.com/). Random GC probes $(40,000)$ were used to monitor hybridization efficiency and four corners fiducial controls (225) were included to assist with overlaying the grid onto the image. Multiple analyses were performed with Limma package in R computing (Wettenhall and Smyth, 2004). The package adopts a linear modeling approach implemented by $\mathrm{lmFit}$ as well as empirical Bayes statistics implemented by eBayes. Genes showing signifi-

Table 1. List and expression levels of receptor like genes highly induced by rice blast fungus

\begin{tabular}{clccccc}
\hline Cluster & \multicolumn{1}{c}{ Putative Function } & Accession No. & R/C_12hpi & R/C_48hpi & S/C_12hpi & S/C_48hpi \\
\hline 1 & Brassinosteroid Insensitive 1-associated receptor & Os11g31540 & 0.811218 & 3.008376 & -0.48823 & -0.51775 \\
& kinase 1 & & & & & \\
12 & Receptor-like protein kinase & Os10g33040 & -0.02861 & 1.910698 & -0.34813 & 0.89525 \\
4 & Receptor-like protein kinase 5 & Os01g53920 & 0.678137 & 1.419601 & -0.28261 & -0.36911 \\
4 & Receptor-like protein kinase 5 & Os06g36270 & 0.448727 & 1.594526 & 0.319967 & -0.83046 \\
4 & Lectin-like receptor kinase 7 & Os03g56160 & 0.320567 & 1.775792 & -0.1518 & -0.13174 \\
4 & TKL_IRAK_DUF26-ld.2 - DUF26 kinases have & Os07g35740 & 0.884713 & 1.265413 & -0.40677 & -0.11206 \\
& homology to DUF26 containing loci & & & & \\
4 & TKL_IRAK_DUF26-lc.4 - DUF26 kinases have & Os07g35700 & 0.158806 & 1.322579 & -0.31056 & -0.0296 \\
& homology to DUF26 containing loci & & & & \\
4 & Cysteine-rich receptor-like protein kinase 21 & Os11g11780 & -0.06805 & 1.32 & 0.384536 & -0.38594 \\
4 & Ser/Thr protein kinase & Os01g04580 & 0.881316 & 1.673645 & -0.27655 & -0.54659 \\
4 & S-locus-like receptor protein kinase & Os01g48000 & 0.978053 & 1.493597 & -0.20809 & -0.47656 \\
4 & MRH1 & Os05g48660 & -0.05179 & 1.115766 & 0.010199 & 0.333886 \\
4 & OsWAK71 - OsWAK receptor-like cytoplasmic & Os07g31190 & 0.587842 & 1.076215 & 0.124959 & 0.165401 \\
& kinase OsWAK-RLCK & & & & \\
4 & Expressed protein & Os06g38970 & -0.00021 & 1.192931 & 0.072643 & -0.40049 \\
4 & Receptor kinase & Os01g05870 & 0.582597 & 1.052183 & 0.045662 & -0.51644 \\
4 & Cysteine-rich receptor-like protein kinase & Os04g56430 & 0.62956 & 2.099881 & -0.2165 & 0.263735 \\
4 & Cysteine-rich repeat secretory protein 55 & Os03g16950 & 1.324019 & 1.504747 & 0.321294 & 0.199844 \\
4 & Cysteine-rich receptor-like protein kinase 12 & Os04g30030 & -0.27925 & 1.126844 & 1.13936 & -0.16362 \\
4 & S-locus-like receptor protein kinase & Os12g03640 & 1.108065 & 0.672518 & -0.23886 & 0.210872 \\
\hline
\end{tabular}

Accession No., Accession number from KOME database (http://cdna01.dna.affrc.go.jp/cDNA/); R/C_12, log2 ratio of gene expression level in incompatible to control; $\mathrm{S} / \mathrm{C}, \log 2$ ratio of gene expression level in compatible to control. 
cantly different expression were selected according to their $\log _{2}$ (Ratio) and $p$-value based on the following criteria: $\log _{2}$ (Ratio) $>=1$ and $p$-value (differentially expressed) $<$ 0.05 . Multivariate statistical tests such as clustering, principal component analysis, and multidimensional scaling were performed with Acuity 3.1 (Axon Instruments). Hierarchical clustering was performed with similarity metrics based on squared Euclidean correlation, and average linkage clustering was used to calculate the distance of genes.

Standard procedures for statistical analysis ( $>2$-fold, $p<0.05$ ) were carried out using the statistical tool of the Microsoft Excel program. We next used the TIGR MultiExperiment Viewer (MeV, http://www.tm4.org/mev.html) to carry out clustering analyses and to generate heat-map expression patterns of the microarray data after mean scaling and $\log _{2}$ transformation.

MapMan analysis. The MapMan program, version 3.1.1, at the Max Plant Institute of Molecular Plant Physiology, Germany (Thimm et al., 2004) was also used for pathway analysis. Genes fold values were transformed to $\log _{2}$ (fold), and then their means were calculated. These non-redundant genes were classified into MapMan BINs and their annotated functions were visualized using the MapMan program by searching against Oryza sativa TIGR5 database.

\section{Results and Discussion}

Validations of samples before transcriptome analysis. Genome wide microarray analysis provides a global view of gene regulation in response to particular biological events. To understand the differential transcriptional regulation of rice in response to rice blast fungus infection, we employed a $300 \mathrm{~K}$ tilling GeneChip in order to investigate RNA changes in the incompatible and compatible type fungal-infected rice leaves. Two different fungal stains, KJ401 and KJ301, which cause resistant and susceptible interactions in rice cv. Jinheung, respectively, were inoculated onto rice leaves. Leaf samples harvested at 12 and 48 hpi showed early induction and high accumulation of PR proteins (Kim et al., 2004; Zhao et al., 2008). Prior to the microarray analysis, we validated $M$. oryzae-infected rice leaves using defense marker genes and phytoalexins (Fig. 1). Expression of rice PR marker genes was tested at 12, 48, and 72 hpi by RT-PCR. All of the tested PR genes, including chitinase 2, barwin, PBZ1, and PR-10, showed accumulation at $12 \mathrm{hpi}$ in incompatible interaction and reached to a peak at 48 hpi (Fig. 1A). Transcriptional differences of these defense marker genes between the in-
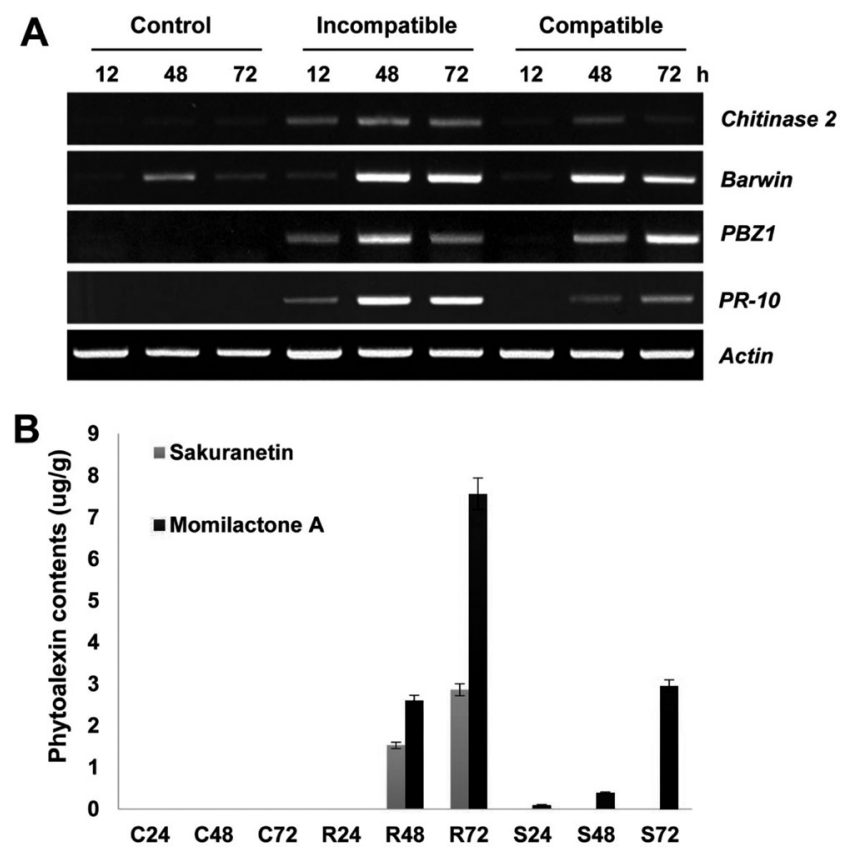

Fig. 1. Validation of samples used for the microarray experiment. (A) Expression profiles of defense marker genes in rice leaves infected with rice blast fungus at indicated time points. (B) Accumulation of phytoalexins, sakuranetin and momilactone $\mathrm{A}$ in fungal infected leaves were detected by HPLC.

compatible and compatible samples were conserved with previous transcriptome and/or proteome results (Kim et al., 2004; Kim et al., 2013). This result suggests that at early time points during rice-rice blast fungus interaction, plant defense-related genes are more rapidly and strongly activated in incompatible type interaction compared to compatible one.

Phytoalexin accumulation is another indication of activation of the plant immune response, and accumulation of phytoalexins was both delayed and reduced in incompatible interaction compared to incompatible one (Hasegawa et al., 2010; Jwa et al., 2006). Two phytoalexins, sakuranetin and momilactone A, were previously found to inhibit $M$. oryzae spore germination in $M$. oryzae-infected leaves (Hasegawa et al., 2010; Jwa et al., 2006; Kodama et al., 1992; Yamane, 2013). Here, the accumulation of sakuranetin and momilactone A was measured in M. oryzae-infected leaves using HPLC-MS/MS. Momilactone A was more highly accumulated in incompatible fungal-infected leaves compared to compatible one at 48 and 72 hpi (Fig. 1B). Sakuranetin was specifically accumulated in incompatible fungal-infected samples (Fig. 1B). Taken together, the expression of PR genes and accumulation of phytoalexins suggests that our samples can be used to investigate transcriptional regula- 
tion during rice and rice blast fungus interaction.

Investigating global changes in gene expression during incompatible and compatible rice-M. oryzae interaction. Differential transcriptional regulation of rice genes in response to fungal pathogen challenge was analyzed, and fold changes were calculated based on gene expression levels in incompatible and compatible type interactions compared with that of control. A total of 608 genes showed significantly increased expression $(>2$-fold, $p<0.05)$ in incompatible or compatible type interactions. A hierarchical clustering (HCL) analysis of all up-regulated genes was generated using multi-experimental viewer (MEV) software after loading $\log _{2}$ fold change values (Fig. 2A) (Cartwright et al., 1981). The expression patterns of incompatible and compatible interactions were different at each time point (Fig. 2A). Among those up-regulated genes, many genes showed increased expression at $12 \mathrm{hpi}$ in incompatible interaction (Fig. 2A).

In a resistance interaction, plant immune signaling was triggered rapidly through the recognition of rice receptors by their elicitors/effectors, and PR genes were more rapidly accumulated in incompatible than in compatible interaction. Transcriptional expression analysis of PR genes also confirmed that an early defense response rapidly occurred in the incompatible interaction at $12 \mathrm{hpi}$, with high accumulation at 48 hpi (Fig. 1A). Therefore, characterization of rapidly or highly accumulated genes in incompatible type interaction is essential to understanding how rice responds to $M$. oryzae infection. Here, we classified those 608 regulated genes into 12 clusters according to their differential expression patterns through $\mathrm{k}$-means clustering installed in MEV (Fig. 2B). Of them, 231 genes in clusters 1, 2, 4, and 12 showed higher accumulation in incompatible interaction than in compatible one (Fig. 2B, Supplementary Tables 2 and 3).

A heat-map of 231 genes associated with incompatible interaction was re-generated (Fig. 3A, Supplementary Tables 2 and 3). As shown in the Fig. 3, clusters 1 and 4 were strongly associated with incompatible interaction at

A
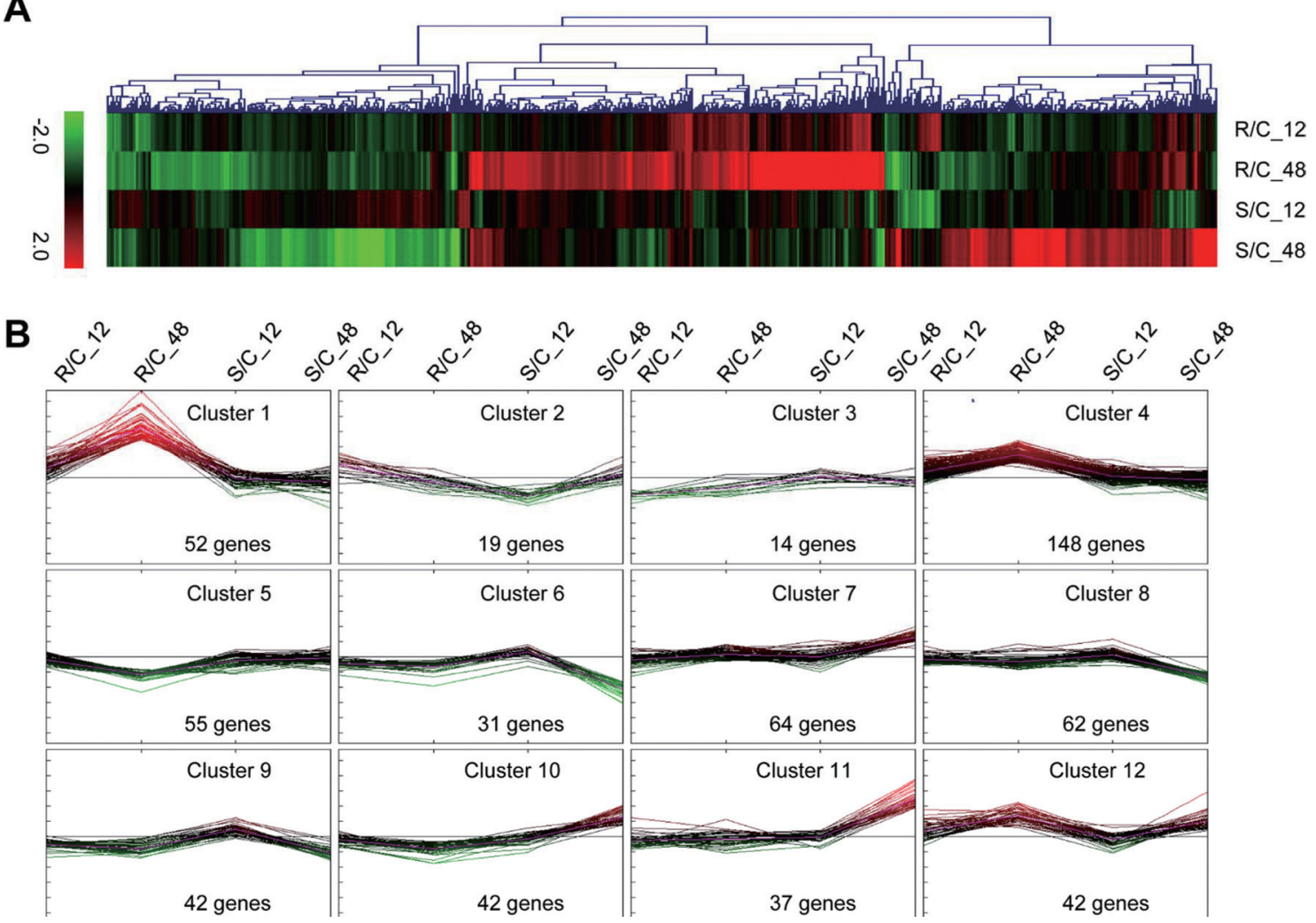

Fig. 2. Analysis of rice transcriptome in response to incompatible (R) and compatible (S) blast fungus infection. (A) Hierarchical clustering and heat-map of gene expression in incompatible and compatible M. oryzae-infected leaf tissues at 12 and 48 hpi. (B) K-mean clustering analysis of gene expression profiles of 608 genes induced by M. oryzae. Acronyms stand for rice gene expression under the following conditions: R/C_12, incompatible sample at $12 \mathrm{hpi}$; R/C_48, incompatible sample at $48 \mathrm{hpi}$; S/C_12, compatible sample at 12 hpi; S/C_48, compatible sample at 48 hpi. 
A
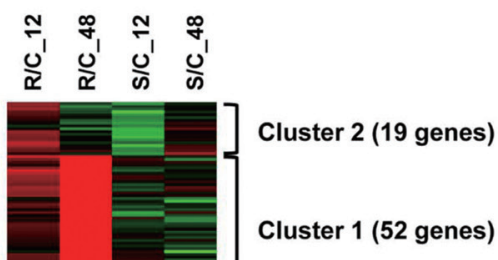

Cluster 1 (52 genes)

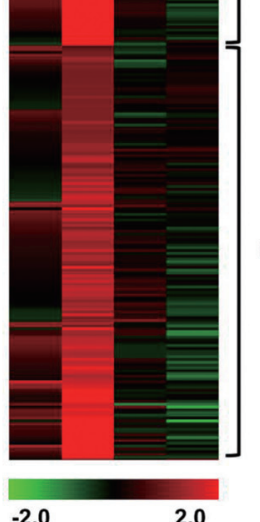

B

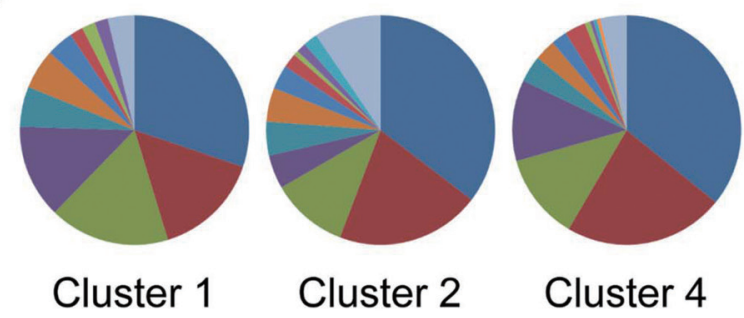

\begin{tabular}{ll|ccc} 
& & & \\
& Cluster 1 & Cluster 2 & Cluster 4 \\
\cline { 2 - 4 } & Metabolic process & $35.37 \%$ & $30.19 \%$ & $35.82 \%$ \\
& Response to stimulus & $20.41 \%$ & $15.09 \%$ & $22.64 \%$ \\
& Cellular process & $10.88 \%$ & $16.98 \%$ & $12.19 \%$ \\
Cluster $\mathbf{4}$ (148 genes) & Biological process & $4.76 \%$ & $13.21 \%$ & $11.44 \%$ \\
& Developmental process & $4.76 \%$ & $5.66 \%$ & $3.73 \%$ \\
& Signaling & $4.76 \%$ & $5.66 \%$ & $2.99 \%$ \\
& Cellular component organization or biogenesis & $3.40 \%$ & $3.77 \%$ & $2.24 \%$ \\
& Establishment of localization & $2.04 \%$ & $1.89 \%$ & $2.99 \%$ \\
& Growth & $0.68 \%$ & $1.89 \%$ & $0.75 \%$ \\
& Multi-organism process & $1.36 \%$ & $1.89 \%$ & $0.50 \%$ \\
& Reproduction & $2.04 \%$ & $0.00 \%$ & $0.50 \%$ \\
& Biological regulation & $0.00 \%$ & $0.00 \%$ & $0.50 \%$ \\
& No Term & $9.52 \%$ & $3.77 \%$ & $3.73 \%$
\end{tabular}

Fig. 3. Heat-Map and Gene Ontology (GO) enrichment analysis of highly expressed genes in incompatible type interaction at 12 and 48 hpi. (A) Heat-Map of 261 highly induced genes, which were classified into four sub-groups based on their expression profiles by Kmean clustering analysis. (B) Number of enriched GO in four sub-groups.

48 hpi (R48) and contained 52 and 148 genes, respectively. The genes belonging to cluster 1 were much more strongly activated at R48 than those in cluster 4 (Fig. 3A). Nineteen genes belonged to cluster 2 , which was highly up-regulated in incompatible interaction at $12 \mathrm{hpi}$ and down-regulated in compatible interaction at $12 \mathrm{hpi}$.

Gene ontology analysis of rice genes associated with the incompatible interaction. To explore the biological processes associated with incompatible interaction, genes in clusters 1,2, and 4 were grouped according to their gene ontology (GO) terms (http://www.gramene.org/), and the enriched GO terms ( $>$ two genes in each GO terms) were analyzed and summarized in Fig. 3B. GO analysis shows that the major GO term associated with incompatible interaction was metabolic process; $35.37 \%, 30.19 \%$, and $35.83 \%$ genes in clusters 1,2 , and 4 , respectively, suggesting that fungal infection may significant alter metabolic processes inside plants. Furthermore, genes related to the responses to stimuli, cell processes, biological processes, development, and signaling showed close association with incompatible interaction in each cluster (Fig. 3B). In the meantime, several genes related to cellular component organization or biogenesis and establishment of localization showed alteration during incompatible interaction. Genes related to biological regulation were only detected in cluster 4 (Fig. 3B). Taken together, our data suggest that rice reprograms metabolic and biological processes to initiate the response to infection by M. oryzae.

Further analysis of these transcriptome data showed that 41 of the 261 incompatible inducing genes were highly expressed at 12 hpi (Supplementary Table 2), whereas 231 were induced at $48 \mathrm{hpi}$ (Supplementary Table 3). At 12 hpi, genes related with oxidative stress (two genes), carbohydrate metabolic processes (three genes), oxidationreduction (five genes), and metabolic processes (six genes) were induced (Fig. 4A). At 48 hpi, genes related with carbohydrate metabolic processes (18 genes), oxidationreduction (23 genes), and metabolic processes (32 genes) were still highly induced (Fig. 4B), suggesting that these cellular processes are essential for rice defense against $M$. oryzae. Moreover, other cellular processes showed involvement in rice defense at $48 \mathrm{hpi}$, such as protein amino acid phosphorylation (19 genes), defense response (13 genes), and biotic stimulus (six genes) (Fig. 4B).

MapMan analysis of rice genes associated with the incompatible interaction. To determine whether or not $M$. oryzae-induced genes are involved in multiple pathways, MapMan analysis (http://mapman.gabipd.org/) was applied to the characterized genes (Thimm et al., 2004). Based on the MapMan biotic stress analysis, the up-regulated genes were classified into different biological processes; three for signaling and one each for ethylene signaling, peroxidases, 


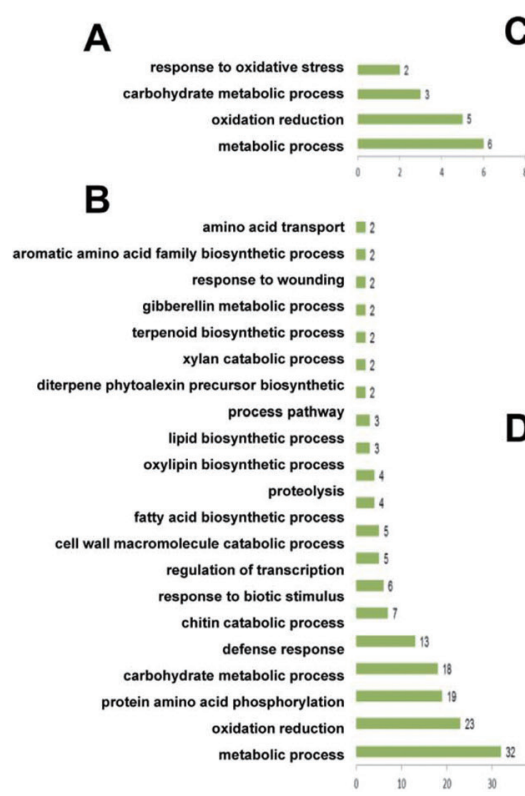

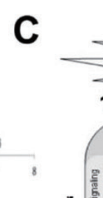

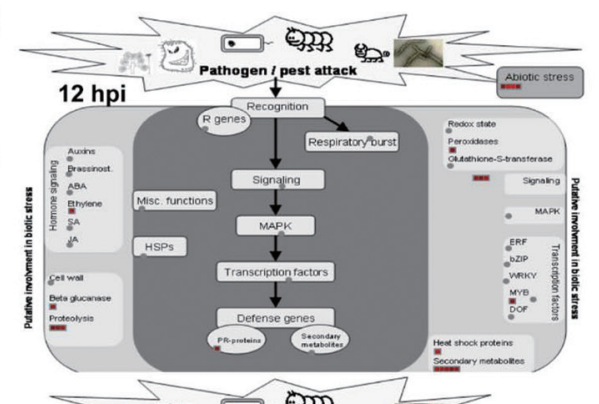

D

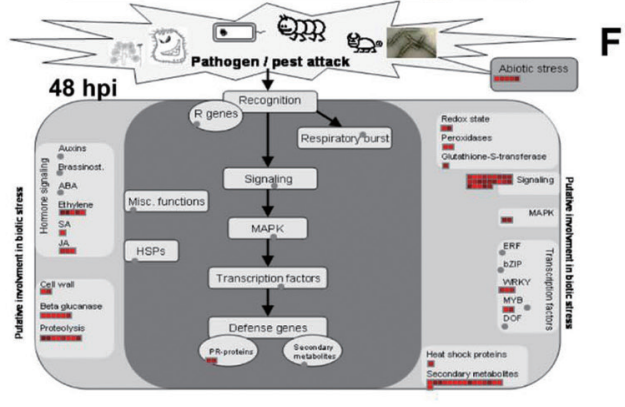

E

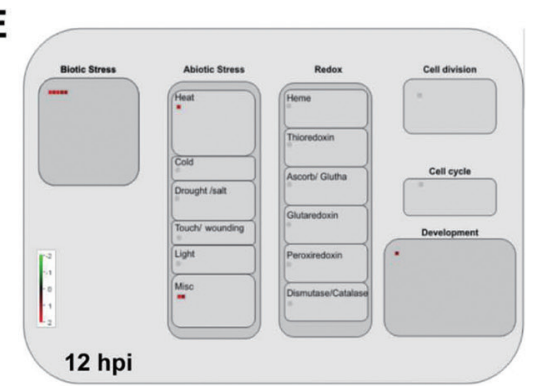

$\mathbf{F}$

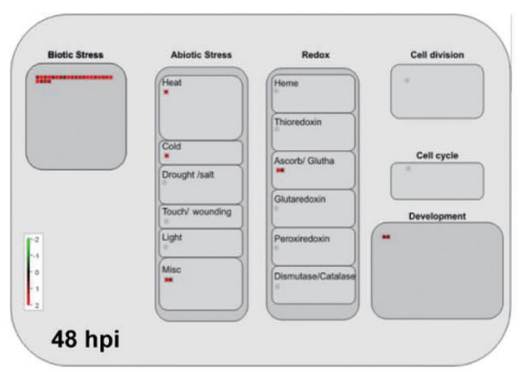

Fig. 4. Gene Ontology (GO) enrichment and MapMan analysis of up-regulated genes in incompatible type fungal infection. Number of enriched GO at 12 (A) and $48 \mathrm{hpi}(\mathrm{B})$. Mapman analysis of up-regulated genes related with biotic stresses at 12 (C) and $48 \mathrm{hpi}$ (D). Regulation overview (E and F) and cellular response overview ( $\mathrm{G}$ and $\mathrm{H})$ of up-regulated genes at 12 and 48 hpi.

myb transcription factor, heat shock, and PR process at 12 hpi (Fig. 4C). At 48 hpi, the up-regulated genes related with biotic stresses showed significantly induction in multiple regulation pathways (Fig. 4D). For hormone signaling, five genes related with ethylene were detected, as well as genes related with salicylic acid (SA) and jasmonic acid (JA) signaling. Previously, exogenous treatment with an ethylene (ET) biosynthesis inhibitor and generator was shown to suppress or induce infection by rice blast fungus in rice (Singh et al., 2004). Incompatible interaction with $M$. oryzae could also activate ET emissions earlier than compatible interaction (Iwai et al., 2006). Taken together, these results suggest that in early fungal infection, ethylene-related pathways may be important for rice innate immunity against rice blast fungus.

Genes related with the cell wall, beta-glucanase, and proteolysis were highly induced in response to $M$. oryzae infection (Fig. 4C and 4D). Cell wall structure and its related degradation enzymes are closely related with plant defense and fungal pathogenicity (Cantu et al., 2008). Overexpression of cell wall degradation enzymes such as Pectin Methylesterase 1 from Arabidopsis have been shown to reduce infection by Botrytis cinerae (Lionetti et al., 2007). Betaglucanases, which digest the beta-1,3-glucan in fungal cell walls, directly inhibit fungal growth (Arlorio et al., 1992). Previous reports also indicated that several rice glucanses are induced in response to $M$. oryzae infection, suggest- ing that expression of glucanases is involved in the antifungal process (Bennett and Wallsgrove, 1994). Secondary metabolites, including terpenes, phenolics, nitrogen and sulfur-containing compounds, play important roles in plant defense against a variety of herbivores and pathogenic microorganisms (Jwa et al., 2006; Ross et al., 2007). Here, several genes related with secondary metabolism were upregulated in response to $M$. oryzae infection, indicating that rice also utilizes secondary metabolites to suppress rice blast fungal infection.

WRKY transcription factors (TFs) are one of the largest families of transcriptional regulators in plants and have DNA-binding ability (Pandey and Somssich, 2009). A total of 109 WRKY TFs exist in rice, and they are related with the immunity response (Ross et al., 2007). In our results, WRKY genes were activated at $48 \mathrm{hpi}$ in incompatible interaction (Fig. 4D, Supplementary Table 3). Previously, OsWRKY45, OsWAKY71, and OsWRKY76 were reported to be involved in $M$. oryzae attack, and OsWRKY45 and OsWRKY76 also activated by Xanthomonas ory$z a e$ infection (Ryu et al., 2006). Overexpression of OsWRKY45 also induces resistance to both $M$. oryzae and $X$. oryzae by mediating benzothiadiazole-inducible blast resistance, which is regulated by SA-dependent defense mechanisms (Shimono et al., 2007; 2012). OsWRKY71 is also induced by chitin oligosaccharide elicitor treatment, and over-expression of OsWRKY71 can trigger expression 
of defense-related genes, such as chitinase family genes. These data suggested that WRKY TFs are essential for defense mechanisms in the early interaction process. MAPK and Myb TFs are also involved in the activation of defense signaling in plants (Ramalingam et al., 2003; Rasmussen et al., 2012). At 48 hpi, several MAPK and Myb TFs as well as signaling-related genes were activated (Fig. 4D, Supplementary Table 3), suggesting that defense signaling-related genes are important to rice defense activation against rice blast fungus.

ROS levels are related with $M$. oryzae infection in rice leaf tissue (Chi et al., 2009; Mittler et al., 2004). Genes related with redox state, peroxidase, and glutathione-Stransferase were more up-regulated at 48 hpi than at $12 \mathrm{hpi}$ (Fig. 4C and 4D, Supplementary Tables 2 and 3), suggesting elevation of ROS in infected tissues. Peroxidase is an ROS-related gene that plays a role in redox balance in cells (Mittler et al., 2004). In rice, peroxidase family genes are activated by infection by various pathogens, such as fungi and bacteria (Hilaire et al., 2001). Consistent with previous reports, two peroxidases were detected in our microarray results, suggesting that ROS signaling is activated in the early stage of $M$. oryzae infections.

A group of PR genes was detected in the microarray results, indicating that rice could rapidly sense $M$. oryzae infection and trigger its defense responses. The PR Bet v I protein family encoding PR-10 homolog genes is the most highly detected PR protein family (Radauer et al., 2008). One of them (PR Bet v I family protein, Os12g36850) was activated at $12 \mathrm{hpi}$, and six different genes were induced at 48 hpi (Supplementary Tables 2 and 3). PR-10 family proteins are activated in response to biotic as well as abiotic stresses (Kim et al., 2004; Kim et al., 2008). In response to M. oryzae infection, PR-10 family genes are differentially expressed in most tissues, such as root, leaf, stem, and flower (Kim et al., 2004; Kim et al., 2008; McGee et al., 2001).

Regulatory and cellular response overviews of those upregulated genes at $12 \mathrm{hpi}$ and $48 \mathrm{hpi}$ were generated by MapMan analysis. Several genes related with protein degradation, modification, TFs, ethylene, receptor kinase, and nutrients were detected at $12 \mathrm{hpi}$, indicating that these processes were important for fungal sensing and/or resistance in early stages (Fig. 4C). Further analysis showed that those genes were also involved in biotic stress, abiotic stress (heat and miscellaneous functions), as well as development processes (Fig. 4E). Regulatory overview at 48 hpi showed that the number of genes with similar functions at $12 \mathrm{hpi}$ increased. These results suggest that molecular processrelated genes are essential for both early and later defense
A

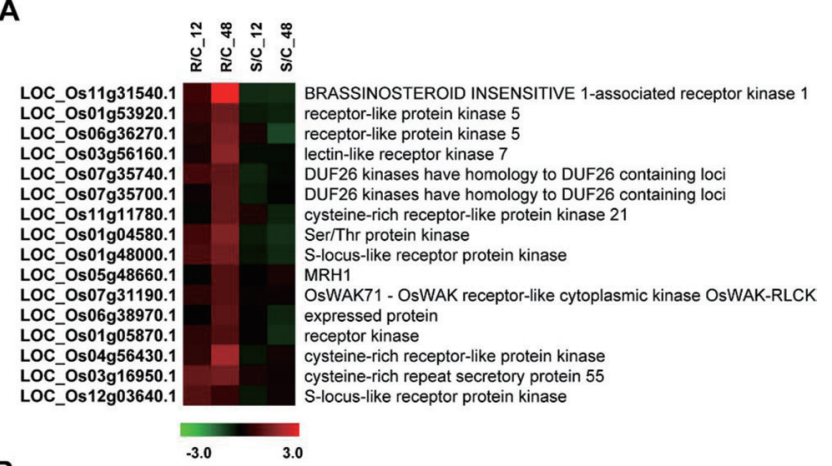

B

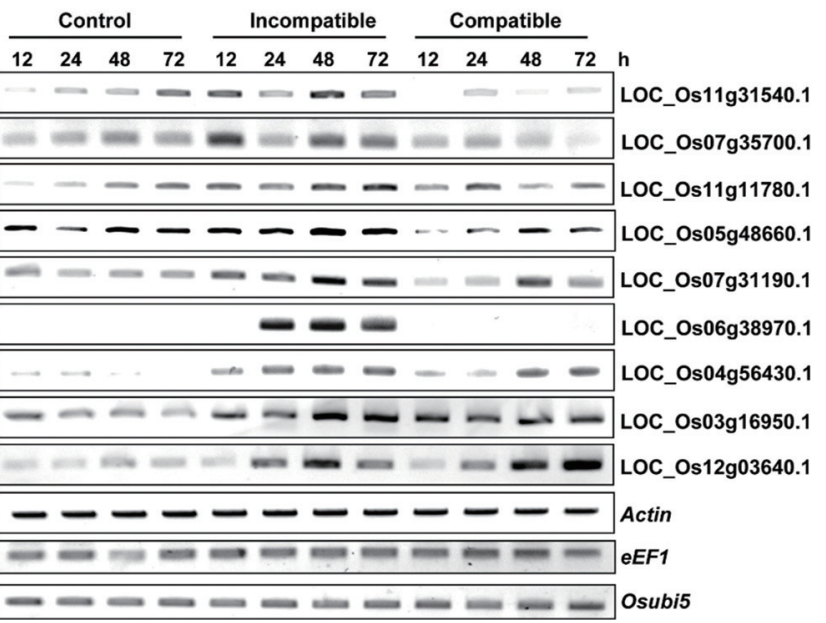

Fig. 5. Up-regulation of receptor-like genes in incompatible type interaction. (A) Heat-map of receptor-like genes identified from microarray analysis. (B) Transcriptional expression of receptorlike genes in response to compatible and incompatible type fungal infections at 12, 24, 48, and 72 hpi were confirmed by RTPCR.

regulation in rice. In addition, several up-regulated genes were newly detected at $48 \mathrm{hpi}$, such as those related with JA and SA signaling, oxidation-reduction, calcium regulation, MAP kinase, and phosphoinositides (Fig. 4F). During rice defense against $M$. oryzae, JA and SA play an essential role in anti-fungal infection (Xie et al., 2011). ROS and calcium signaling are also essential for defense activation (Chi et al., 2009; Lecourieux et al., 2006; Mittler et al., 2004). These reports are consistent with our transcriptome results. For the cellular response overview, the number of up-regulated genes related with biotic stresses increased, and genes related with oxidation-reduction were newly detected (Fig. 4C and 4D). Taken together, our finding of upregulated genes in response to $M$. oryzae infection provides an insight into rice-rice blast fungus interaction.

Receptor-like kinase genes induced by $M$. oryzae infection. Plant genomes encode a large number of RLKs 
involved in signal transduction during plant development and innate immunity (Greeff et al., 2012; Steinwand and Kieber, 2010). During defense, those RLKs recognize pathogen-associated signals and trigger a broad range of downstream defense responses (Jwa et al., 2006). Therefore, study of RLKs involved in defense signaling is essential for understanding host counterattack processes. Here, 18 rice RLKs showed significant up-regulation in incompatible interaction compared to compatible one (Fig. 5A), suggesting those RLKs may be essential for the rice antifungal process (Table 1).

A rice brassinosteroid insensitive 1-associated receptor kinase 1 (BAK1) showed specific expression in incompatible interaction at both 12 and 48 hpi (Fig. 5A). In Arabidopsis, BAK1 is a co-receptor of FLS2 and EFR, and it mediates pattern recognition receptor (PRR)-dependent signaling to initiate innate immunity (Jones and Dangl, 2006). These data suggested that rice BAK1 may also be related with recognition of PRR signaling from rice blast fungus to mediate activation of defense responses. Cysteine-rich receptor-like protein kinase is also known to be involved in defense signal transduction in Arabidopsis (Ederli et al., 2011). DUF26 (Domain of Unknown Function 26) also belongs to the cysteine-rich RLK (CRK) family. DUF26 has been suggested to play an important role in defense against pathogen infection. Overexpression of CRKs could enhance plant resistance to pathogen infection by triggering hypersensitive responses (Acharya et al., 2007; Chen et al., 2003; Chen et al., 2004). In rice, DUF26 protein is highly enriched in the apoplastic region in response to infection by $M$. oryzae and X. oryzae (Kim et al., 2004; Wang et al., 2013), indicating that the DUF26 gene is involved in pathogen resistance in rice. In our study, six CRKs, including two DUF26 kinases, were induced in the incompatible responses (Fig. 5A), indicating that activation of those genes are involved in resistance to rice blast fungus infection.

The plasma membrane localized wall-associated kinase (WAK) family of proteins is tightly bound to plant cell walls (He et al., 1996), WAK proteins have the ability to recognize oligogalacturonides released from the cell wall during pathogen infection, known as damage-associated molecular patterns (DAMPs), and trigger innate immune responses (Brutus et al., 2010). Overexpression of WAK1 in Arabidopsis leads to increased resistance to the necrotrophic fungus Botrytis cinerea. In rice, overexpression of OsWAK1 increases resistance to $M$. oryzae infection. Here, OsWAKY71 was highly accumulated in response to incompatible type fungal infection (Fig. 5A). These data suggest that OsWAK71 may play a role in anti-fungal in- fection through DAMP-triggered immunity.

Two TKL (tyrosine kinase-like)-IRAK (interleukin-1 receptor-associated kinase) type RLKs were also induced by incompatible type interaction (Fig. 5A). TKL-IRAKtype RLKs constitute a conserved protein family that is present in both animal and plants, and they are related with development, stress, symbiosis, as well as innate immunity (Dardick and Ronald, 2006). TKL-IRAK RLKs are also related with PRR-mediated defense signaling in plants, such as rice XA21 and Arabidopsis FLS2 (Dardick and Ronald, 2006; Dunning et al., 2007). Taken together, our transcriptome results indicate that the RLKs highly detected in incompatible interaction may be involved in the recognition of fungal elicitors/effectors to trigger host immune responses.

To further verify these results, semi-quantitative RT-PCR analysis was carried out on six identified RLKs (Fig. 5B). Rice actin, eEF1, and Osubi5 were employed as internal standards (Fig. 5B). Eight RLK genes were selected to confirm transcriptional expression in compatible and incompatible type interactions at $12,24,48$, and 72 hpi. Compared with their expression levels in uninfected (control) and compatible type fungal-infected leaves, all of the genes were highly expressed in incompatible type interaction (Fig. 5), indicating good correlation with our transcriptome data. Further functional analyses of these candidate genes are required for the development of rice plants with enhanced defense responses against rice blast fungus infection.

\section{Conclusions}

In this study, we performed transcriptome analysis using a microarray to study the responses of rice cells in the early stage of $M$. oryzae infection. A total number of 608 genes were differentially expressed in response to compatible and incompatible type M. oryzae infections. Among them, 231 genes were more highly accumulated in incompatible type interaction compared to compatible one, indicating those genes play a major role in the response against fungal infection. Most of those genes functioned in the metabolic process, response to stimuli, and cellular processes, indicating these processes are strongly altered by fungal infection. The biotic stress map view of MapMan analysis showed that genes related with signaling (WRKY and Myb transcription factors) and secondary metabolism were rapidly induced, and ethylene was important for the defense response. Moreover, a total of 18 receptor-like protein kinase genes were identified in our results, and their expression was validated by RT-PCR analysis. Thus, RLK genes may be essential for the sensing of pathogen signals and induc- 
tion of immunity in rice against rice blast fungus infection.

\section{Acknowledgments}

This work was supported by the Basic Science Research Program through the National Research Foundation of Korea (NRF) funded by the Ministry of Education, Science, and Technology (2013R1A1A1A05005407), and NextGeneration BioGreen 21 Program (Plant Molecular Breeding Center, PJ008021), Rural Development Administration, Republic of Korea.

\section{References}

Acharya, B. R., Raina, S., Maqbool, S. B., Jagadeeswaran, G., Mosher, S. L., Appel, H. M., Schultz, J. C., Klessig, D. F. and Raina, R. 2007. Overexpression of CRK13, an Arabidopsis cysteine-rich receptor-like kinase, results in enhanced resistance to Pseudomonas syringae. Plant J. 50:488-499.

Arlorio, M., Ludwig, A., Boller, T. and Bonfante, P. 1992. Inhibition of fungal growth by plant chitinases and $\beta-1,3-$ glucanases. Protoplasma 171:34-43.

Bagnaresi, P., Biselli, C., Orru, L., Urso, S., Crispino, L., Abbruscato, P., Piffanelli, P., Lupotto, E., Cattivelli, L. and Vale, G. 2012. Comparative transcriptome profiling of the early response to Magnaporthe oryzae in durable resistant vs susceptible rice (Oryza sativa L.) genotypes. PLoS One 7:e51609.

Bennett, R. N. and Wallsgrove, R. M. 1994. Secondary metabolites in plant defence mechanisms. New Phytol. 127:617-633.

Brutus, A., Sicilia, F., Macone, A., Cervone, F. and De Lorenzo, G. 2010. A domain swap approach reveals a role of the plant wall-associated kinase 1 (WAK1) as a receptor of oligogalacturonides. Proc. Natl. Acad. Sci. USA 107:9452-9457.

Cantu, D., Vicente, A. R., Labavitch, J. M., Bennett, A. B. and Powell, A. L. 2008. Strangers in the matrix: Plant cell walls and pathogen susceptibility. Trends Plant Sci. 13:610-617.

Cartwright, D. W., Langcake, P., Pryce, R. J., Leworthy, D. P. and Ride, J. P. 1981. Isolation and characterization of two phytoalexins from rice as momilactones A and B. Phytochemistry 20:535-537.

Chen, K., Du, L. and Chen, Z. 2003. Sensitization of defense responses and activation of programmed cell death by a pathogen-induced receptor-like protein kinase in Arabidopsis. Plant Mol. Biol. 53:61-74.

Chen, K., Fan, B., Du, L. and Chen, Z. 2004. Activation of hypersensitive cell death by pathogen-induced receptor-like protein kinases from arabidopsis. Plant Mol. Biol. 56:271-283.

Chi, M. H., Park, S. Y., Kim, S. and Lee, Y. H. 2009. A novel pathogenicity gene is required in the rice blast fungus to suppress the basal defenses of the host. PLoS Pathog. 5:e1000401.

Chinchilla, D., Zipfel, C., Robatzek, S., Kemmerling, B., Nurnberger, T., Jones, J. D., Felix, G. and Boller, T. 2007. A flagel- lin-induced complex of the receptor FLS2 and BAK1 initiates plant defence. Nature 448:497-500.

Couch, B. C., Fudal, I., Lebrun, M. H., Tharreau, D., Valent, B., van Kim, P., Notteghem, J. L. and Kohn, L. M. 2005. Origins of host-specific populations of the blast pathogen Magnaporthe oryzae in crop domestication with subsequent expansion of pandemic clones on rice and weeds of rice. Genetics 170:613-630.

Dardick, C. and Ronald, P. 2006. Plant and animal pathogen recognition receptors signal through non-RD kinases. PLoS Pathog. 2:e2.

Datta, K., Velazhahan, R., Oliva, N., Ona, I., Mew, T., Khush, G.,S., Muthukrishnan, S. and Datta, S.,K. 1999. Over-expression of the cloned rice thaumatin-like protein (PR-5) gene in transgenic rice plants enhances environmental friendly resistance to rhizoctonia solani causing sheath blight disease. Theor. Appl. Genet. 98:1138-1145.

Dunning, F. M., Sun, W., Jansen, K. L., Helft, L. and Bent, A. F. 2007. Identification and mutational analysis of Arabidopsis FLS2 leucine-rich repeat domain residues that contribute to flagellin perception. Plant Cell 19:3297-3313.

Ederli, L., Madeo, L., Calderini, O., Gehring, C., Moretti, C., Buonaurio, R., Paolocci, F. and Pasqualini, S. 2011. The Arabidopsis thaliana cysteine-rich receptor-like kinase CRK20 modulates host responses to Pseudomonas syringae pv. tomato DC3000 infection. J. Plant Physiol. 168:1784-1794.

Frederickson Matika, D. E. and Loake, G. J. 2014. Redox regulation in plant immune function. Antioxid. Redox Signal. doi:10.1089/ars.2013.5679.

Greeff, C., Roux, M., Mundy, J. and Petersen, M. 2012. Receptorlike kinase complexes in plant innate immunity. Front. Plant. Sci. 3:209.

Hasegawa, M., Mitsuhara, I., Seo, S., Imai, T., Koga, J., Okada, K., Yamane, H. and Ohashi, Y. 2010. Phytoalexin accumulation in the interaction between rice and the blast fungus. Mol. Plant-Microbe Interact. 23:1000-1011.

He, Z. H., Fujiki, M. and Kohorn, B. D. 1996. A cell wall-associated, receptor-like protein kinase. J. Biol. Chem. 271:1978919793.

Hilaire, E., Young, S. A., Willard, L. H., McGee, J. D., Sweat, T., Chittoor, J. M., Guikema, J. A. and Leach, J. E. 2001. Vascular defense responses in rice: Peroxidase accumulation in xylem parenchyma cells and xylem wall thickening. Mol. PlantMicrobe Interact. 14:1411-1419.

Iqbal, M. M., Nazir, F., Ali, S., Asif, M. A., Zafar, Y., Iqbal, J. and Ali, G. M. 2012. Over expression of rice chitinase gene in transgenic peanut (Arachis hypogaea L.) improves resistance against leaf spot. Mol. Biotechnol. 50:129-136.

Iwai, T., Miyasaka, A., Seo, S. and Ohashi, Y. 2006. Contribution of ethylene biosynthesis for resistance to blast fungus infection in young rice plants. Plant Physiol. 142:1202-1215.

Jones, J. D. and Dangl, J. L. 2006. The plant immune system. Nature 444:323-329.

Jwa, N. S., Agrawal, G. K., Tamogami, S., Yonekura, M., Han, O., 
Iwahashi, H. and Rakwal, R. 2006. Role of defense/stressrelated marker genes, proteins and secondary metabolites in defining rice self-defense mechanisms. Plant Physiol. Biochem. 44:261-273.

Kawahara, Y., Oono, Y., Kanamori, H., Matsumoto, T., Itoh, T. and Minami, E. 2012. Simultaneous RNA-seq analysis of a mixed transcriptome of rice and blast fungus interaction. PLoS One 7:e49423.

Kim, S. G., Wang, Y., Lee, K. H., Park, Z. Y., Park, J., Wu, J., Kwon, S. J., Lee, Y. H., Agrawal, G. K., Rakwal, R., Kim, S. T. and Kang, K. Y. 2013. In-depth insight into in vivo apoplastic secretome of rice-Magnaporthe oryzae interaction. J. Proteomics 78:58-71.

Kim, S. T., Kim, S. G., Hwang, D. H., Kang, S. Y., Kim, H. J., Lee, B. H., Lee, J. J. and Kang, K. Y. 2004. Proteomic analysis of pathogen-responsive proteins from rice leaves induced by rice blast fungus, Magnaporthe grisea. Proteomics 4:3569-3578.

Kim, S. T., Yu, S., Kang, Y. H., Kim, S. G., Kim, J. Y., Kim, S. H. and Kang, K. Y. 2008. The rice pathogen-related protein 10 (JIOsPR 10) is induced by abiotic and biotic stresses and exhibits ribonuclease activity. Plant Cell Rep. 27:593-603.

Kim, S. T., Kang, Y. H., Wang, Y., Wu, J., Park, Z. Y., Rakwal, R., Agrawal, G. K., Lee, S. Y. and Kang, K. Y. 2009. Secretome analysis of differentially induced proteins in rice suspensioncultured cells triggered by rice blast fungus and elicitor. Proteomics 9:1302-1313.

Kodama, O., Miyakawa, J., Akatsuka, T. and Kiyosawa, S. 1992. Sakuranetin, a flavanone phytoalexin from ultraviolet-irradiated rice leaves. Phytochemistry 31:3807-3809.

Lecourieux, D., Ranjeva, R. and Pugin, A. 2006. Calcium in plant defence-signalling pathways. New Phytol. 171:249-269.

Lionetti, V., Raiola, A., Camardella, L., Giovane, A., Obel, N., Pauly, M., Favaron, F., Cervone, F. and Bellincampi, D. 2007. Overexpression of pectin methylesterase inhibitors in Arabidopsis restricts fungal infection by Botrytis cinerea. Plant Physiol. 143:1871-1880.

McGee, J. D., Hamer, J. E. and Hodges, T. K. 2001. Characterization of a PR-10 pathogenesis-related gene family induced in rice during infection with Magnaporthe grisea. Mol. PlantMicrobe Interact. 14:877-886.

Mittler, R., Vanderauwera, S., Gollery, M. and Van Breusegem, F. 2004. Reactive oxygen gene network of plants. Trends Plant Sci. 9:490-498.

Pandey, S. P. and Somssich, I. E. 2009. The role of WRKY transcription factors in plant immunity. Plant Physiol. 150:16481655.

Pang, C. H., Li, K. and Wang, B. 2011. Overexpression of SsCHLAPXs confers protection against oxidative stress induced by high light in transgenic Arabidopsis thaliana. Physiol. Plant. 143:355-366.

Radauer, C., Lackner, P. and Breiteneder, H. 2008. The bet v 1 fold: An ancient, versatile scaffold for binding of large, hydrophobic ligands. BMC Evol. Biol. 8:286.
Ramalingam, J., Vera Cruz, C. M., Kukreja, K., Chittoor, J. M., Wu, J. L., Lee, S. W., Baraoidan, M., George, M. L., Cohen, M. B., Hulbert, S. H., Leach, J. E. and Leung, H. 2003. Candidate defense genes from rice, barley, and maize and their association with qualitative and quantitative resistance in rice. Mol. Plant-Microbe Interact. 16:14-24.

Rasmussen, M. W., Roux, M., Petersen, M. and Mundy, J. 2012. MAP kinase cascades in Arabidopsis innate immunity. Front. Plant. Sci. 3:169.

Ribot, C., Hirsch, J., Balzergue, S., Tharreau, D., Notteghem, J. L., Lebrun, M. H. and Morel, J. B. 2008. Susceptibility of rice to the blast fungus, Magnaporthe grisea. J. Plant Physiol. 165:114-124.

Ross, C. A., Liu, Y. and Shen, Q. J. 2007. The WRKY gene family in rice (Oryza sativa). J. Integr. Plant Biol. 49:827-842.

Ryu, H. S., Han, M., Lee, S. K., Cho, J. I., Ryoo, N., Heu, S., Lee, Y. H., Bhoo, S. H., Wang, G. L., Hahn, T. R. and Jeon, J. S. 2006. A comprehensive expression analysis of the WRKY gene superfamily in rice plants during defense response. Plant Cell Rep. 25:836-847.

Sels, J., Mathys, J., De Coninck, B. M., Cammue, B. P. and De Bolle, M. F. 2008. Plant pathogenesis-related (PR) proteins: A focus on PR peptides. Plant Physiol. Biochem. 46:941-950.

Shimono, M., Koga, H., Akagi, A., Hayashi, N., Goto, S., Sawada, M., Kurihara, T., Matsushita, A., Sugano, S., Jiang, C. J., Kaku, H., Inoue, H. and Takatsuji, H. 2012. Rice WRKY45 plays important roles in fungal and bacterial disease resistance. Mol. Plant. Pathol. 13:83-94.

Shimono, M., Sugano, S., Nakayama, A., Jiang, C. J., Ono, K., Toki, S. and Takatsuji, H. 2007. Rice WRKY45 plays a crucial role in benzothiadiazole-inducible blast resistance. Plant Cell. 19:2064-2076.

Singh, M. P., Lee, F. N., Counce, P. A. and Gibbons, J. H. 2004. Mediation of partial resistance to rice blast through anaerobic induction of ethylene. Phytopathology 94:819-825.

Steinwand, B. J. and Kieber, J. J. 2010. The role of receptorlike kinases in regulating cell wall function. Plant Physiol. 153:479-484.

Talbot, N. J. 2003. On the trail of a cereal killer: Exploring the biology of Magnaporthe grisea. Annu. Rev. Microbiol. 57:177202.

Tamogami, S. and Kodama, O. 2000. Coronatine elicits phytoalexin production in rice leaves (Oryza sativa L.) in the same manner as jasmonic acid. Phytochemistry 54:689-694.

Thimm, O., Blasing, O., Gibon, Y., Nagel, A., Meyer, S., Kruger, P., Selbig, J., Muller, L. A., Rhee, S. Y. and Stitt, M. 2004. MAPMAN: A user-driven tool to display genomics data sets onto diagrams of metabolic pathways and other biological processes. Plant J. 37:914-939.

Wang, Y., Kim, S. G., Wu, J., Huh, H. H., Lee, S. J., Rakwal, R., Agrawal, G. K., Park, Z. Y., Kang, K. Y. and Kim, S. T. 2013. Secretome analysis of the rice bacterium Xanthomonas oryzae (Xoo) using in vitro and in planta systems. Proteomics 13:1901-1912. 
Wei, T., Ou, B., Li, J., Zhao, Y., Guo, D., Zhu, Y., Chen, Z., Gu, H., Li, C., Qin, G. and Qu, L. J. 2013. Transcriptional profiling of rice early response to Magnaporthe oryzae identified OsWRKYs as important regulators in rice blast resistance. PLoS One. 8:e59720.

Wettenhall, J. M. and Smyth, G. K. 2004. limmaGUI: A graphical user interface for linear modeling of microarray data. Bioinformatics 20:3705-3706.

Wu, J., Wang, Y., Kim, S. T., Kim, S. G. and Kang, K. Y. 2013. Characterization of a newly identified rice chitinase-like protein (OsCLP) homologous to xylanase inhibitor. BMC Biotechnol. 13:4.
Xie, X. Z., Xue, Y. J., Zhou, J. J., Zhang, B., Chang, H. and Takano, M. 2011. Phytochromes regulate SA and JA signaling pathways in rice and are required for developmentally controlled resistance to Magnaporthe grisea. Mol. Plant. 4:688-696.

Yamane, H. 2013. Biosynthesis of phytoalexins and regulatory mechanisms of it in rice. Biosci. Biotechnol. Biochem. 77:1141-1148.

Zhao, C. J., Wang, A. R., Shi, Y. J., Wang, L. Q., Liu, W. D., Wang, Z. H. and Lu, G. D. 2008. Identification of defenserelated genes in rice responding to challenge by Rhizoctonia solani. Theor. Appl. Genet. 116:501-516. 